\title{
The Assembly of the First Galaxies
}

\author{
Zoltán Haiman ${ }^{1,2}$ \\ 1 Princeton University Observatory, Peyton Hall, 08544 NJ, USA \\ 2 Hubble Fellow
}

\begin{abstract}
The first galaxies formed at high redshifts, and were likely substantially less massive than typical galaxies in the local universe. We argue that (1) the reionization of a clumpy intergalactic medium (IGM) by redshift $z \approx 6,(2)$ its enrichment by metals by $z \approx 3$ without disturbing the Ly $\alpha$ forest, and (3) the presence of supermassive black holes powering the recently discovered bright quasars at $z \sim 6$, strongly suggest that a population of low-mass galaxies exists beyond redshifts $z \gtrsim 6$. Although the first stars could have been born in dark matter halos with virial temperatures as low as $T_{\text {vir }} \approx 200 \mathrm{~K}$, collapsing as early as $z \sim 25$, the first galaxies likely appeared in significant numbers only in halos with $T_{\text {vir }}>10^{4} \mathrm{~K}$ that collapsed later $(z \sim 15)$. The gas in these more massive halos initially contracts isothermally to high densities by atomic Ly $\alpha$ cooling. $\mathrm{H}_{2}$ molecules can then form efficiently via non-equilibrium gas-phase chemistry, allowing the gas to cool further to $T \sim 100 \mathrm{~K}$, and fragment on stellar mass scales. These halos can harbor the first generation of "mini-galaxies" that reionized the universe. The continuum and line emission from these sources, as well as their Ly $\alpha$ cooling radiation, can be detected in the future by NGST and other instruments.
\end{abstract}

\section{Introduction}

Recent measurements of the cosmic microwave background (CMB) temperature anisotropies, determinations of the luminosity distance to distant type Ia Supernovae (SNe), and other observations have led to the emergence of a robust "best-fit" cosmological model with energy densities in cold dark matter (CDM) and "dark energy" of $\left(\Omega_{\mathrm{M}}, \Omega_{\Lambda}\right) \approx(0.3,0.7)$. The growth of density fluctuations, and their evolution into non-linear dark matter structures can be followed in detail from first principles by semi-analytic methods [1.2] and $\mathrm{N}$-body simulations [3]. Structure formation is "bottom-up", with low-mass halos condensing first. Halos with the masses of globular clusters, $10^{5-6} \mathrm{M}_{\odot}$, are predicted to have condensed as early as $\sim 1 \%$ of the current age of the universe, or redshift $z \sim 25$. It is natural to identify these condensations as the sites where the first astrophysical objects, such as stars, or quasars, were born.

\section{Current Evidence for High Redshift Galaxies}

Current observations directly probe the universe out to redshift $z \sim 6$, with the record-holder quasar at $z=6.28$ [ [ 4 , and the history of star-formation and of quasar activity mapped out to $z \sim 5$ [5]. However, there is convincing 


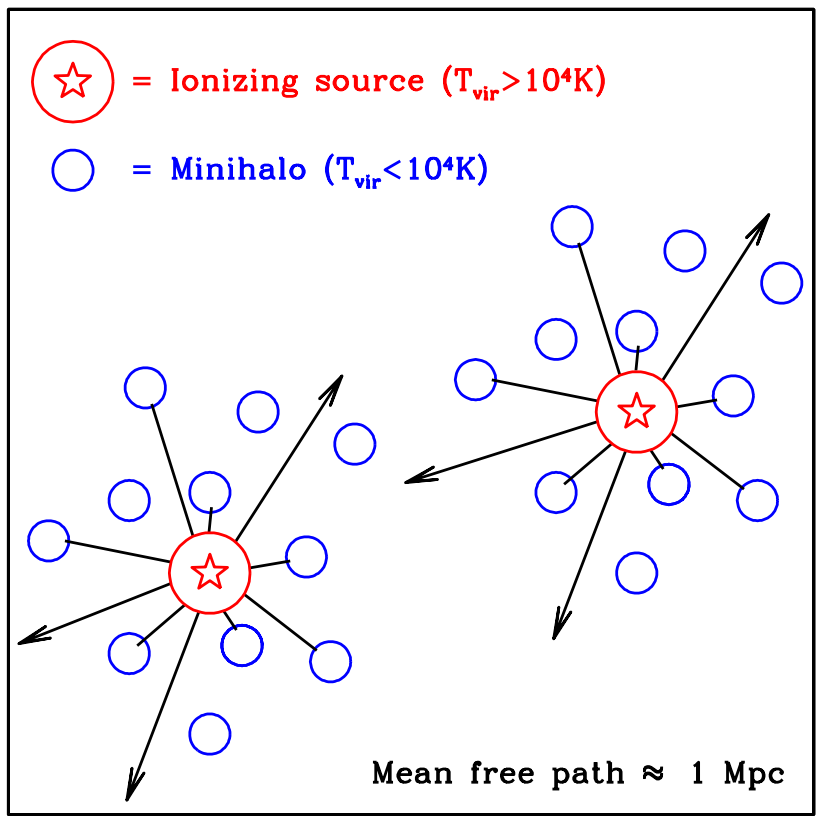

Fig. 1. Dense minihalos with virial temperatures $10^{3} \mathrm{~K} \lesssim T_{\text {vir }} \lesssim 10^{4} \mathrm{~K}$ are an important sink of ionizing radiation as they surround UV sources at high redshifts $(z \gtrsim 6)$. As a population, such minihalos raise by about an order of magnitude the budget of ionizing photons necessary to fully reionize the universe by redshift $z \approx 6$ [6].

observational evidence that an additional, yet undiscovered population of lowmass galaxies exists at these redshifts and beyond.

\subsection{Reionization of a Clumpy Universe}

The lack of a Gunn-Peterson trough in the spectra of all quasars to date, except for the record holder quasar at $z=6.28$, imply that the universe is highly ionized prior to redshift $z \sim 6$ (perhaps near $z \sim 6.3$ ). An early population of stars or quasars could have photoionized the IGM, if they produced at least one ionizing photon per hydrogen atom in the universe. A naive extrapolation of the luminosity density of bright quasars towards $z=6$ reveals that these sources fall short of this requirement. Extrapolating the known population of Lyman Break Galaxies (LBGs) towards $z=6$ comes closer: assuming that $15 \%$ of the ionizing radiation from LBGs escapes into the IGM (on average, relative to the escape fraction at $1500 \AA$ ), a naive extrapolation shows that LBGs emitted $\sim$ one ionizing photon per hydrogen atom prior to $z=6[6]$. Although this 
would be sufficient to ionize every $\mathrm{H}$ atom once, the required photon budget exceeds this value. The earliest ionizing sources are likely surrounded by numerous "minihalos" that had collapsed earlier, but had failed to cool and form any stars or quasars This is illustrated in Figure 11. The minihalos have typical masses below $10^{7} \mathrm{M}_{\odot}$, and represent a population of dense clumps that is currently unresolved in large three-dimensional cosmological simulations. The UV radiation incident on the minihalos heats their gas to $T \approx 10^{4} \mathrm{~K}$, causing it to photo-evaporate [7,8]. The mean free path of ionizing photons, before they are absorbed by an evaporating minihalo, is about $\sim 1$ (comoving) Mpc. As a result, the typical fate of an ionizing photon, emitted at $z \gtrsim 6$, is to be absorbed by a minihalo within a small fraction of the Hubble distance - before it could contribute to the reionization of the bulk of the IGM. A simple model of the photoevaporation process [6], summed over the expected population of minihalos, reveals that on average, an $\mathrm{H}$ atom in the universe recombines $\gtrsim 10$ times before redshift $z=6$. The implication is that the ionizing emissivity at $z>6$ was $\sim 10$ times higher than provided by a straightforward extrapolation back in time of known quasar and galaxy populations.

\subsection{Metal Enrichment of the Intergalactic Medium}

Recent detections of CIV and SIV absorption associated with low column density Ly $\alpha$ absorption lines in the spectra of distant quasars imply that the universe was enriched by metals to a mean level of $\sim 10^{-3} Z_{\odot}$ prior to redshift $z \sim 3$. These absorbers, whose HI column densities are as low as $10^{14.2} \mathrm{~cm}^{-2}$ [9], are identified in cosmological hydrodynamical simulations as regions with typical densities only a few times above that of the mean IGM. This low density rules out "in-situ" metal enrichment, and raises the question: where did these metals come from? LBGs discovered at redshifts $z=3-4$ would be natural candidates for producing and dispersing heavy elements in galactic winds [10]. In order for a significant fraction of the volume to be enriched within a Hubble time, the winds need to move heavy elements at mean speeds close to $1000 \mathrm{~km} / \mathrm{s}$ (the typical separation of LBGs is a few Mpc). While outflows at such high speeds are known to occur, it is unclear whether this scenario can be reconciled with the observed line-widths of the absorbers, which are as narrow as $\sim 20 \mathrm{~km} / \mathrm{s}$, when the cooling time in the low-density IGM is exceedingly long at $z \sim 3$. An alternative possibility is early enrichment by much more numerous low-mass systems, with typical separations of $<0.1 \mathrm{Mpc}$, which can drive outflows at their escape velocities of a few $\times 10 \mathrm{~km} / \mathrm{s}$ (see also 11]). In Figure 2, we show the maximum masses of halos (using the halo mass function from [3]) whose mean separation is small enough so that their distance can be crossed in the age of the universe (at each redshift) at speeds of 10, 100, or $1000 \mathrm{~km} / \mathrm{s}$. In all three cases, the shaded regions correspond to requiring that the dispersed metals fill $3-100 \%$ of the volume. The figure reveals that at $z \approx 3-4$, systems with

\footnotetext{
${ }^{1}$ If minihalos are themselves the sources of ionizing radiation, and their gas is photoevaporated "inside-out", then the required photon budget is likely to be even higher.
} 


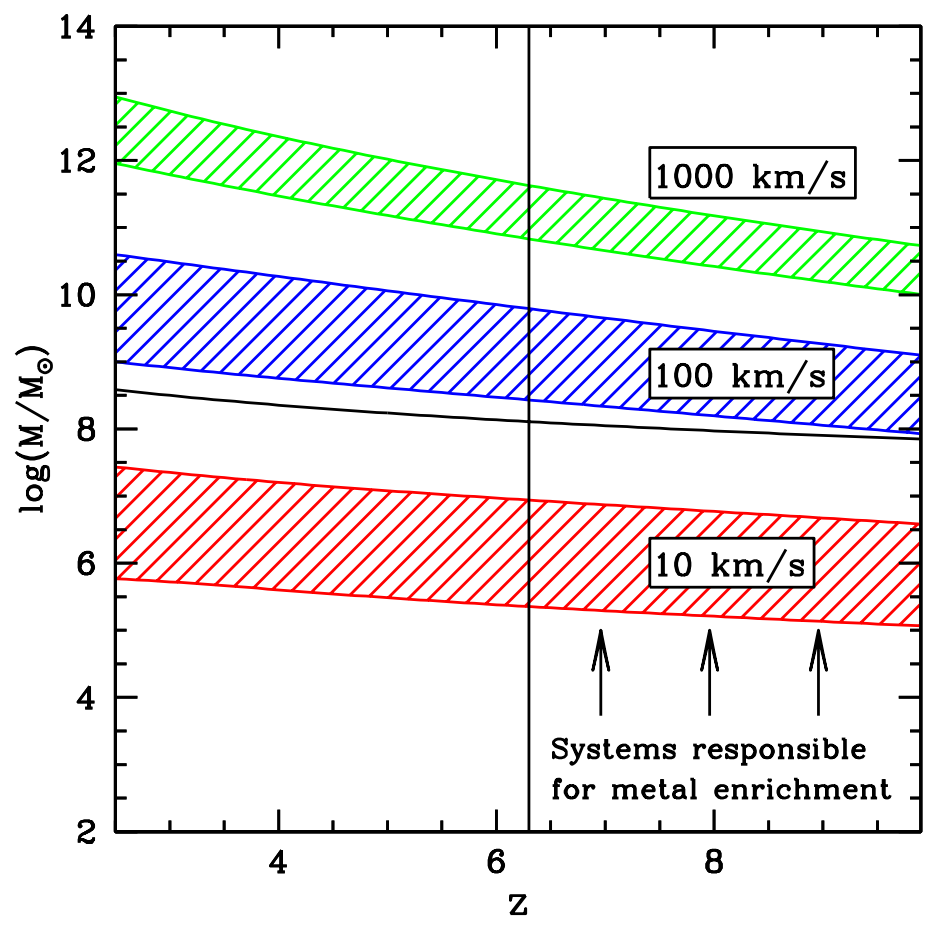

Fig. 2. Typical masses of halos responsible for dispersing metals into the IGM if metals travel at mean speeds of 10, 100, or $1000 \mathrm{~km} / \mathrm{s}$. The upper (lower) envelope for each shaded curve corresponds to the requirement that $3(100) \%$ of the volume is enriched within the age of the universe at redshift $z$. The solid horizontal curve shows the halo that has a velocity dispersion of $\sigma=10 \mathrm{~km} / \mathrm{s}$; the vertical line shows the minimum reionization redshift at $z=6.3$. Widely dispersing the metals at low $(\sim 10 \mathrm{~km} / \mathrm{s})$ speeds requires enrichment by low-mass systems, $M \approx 10^{6} \mathrm{M}_{\odot}$, which produce metals only prior to reionization.

$M \approx 10^{12} \mathrm{M}_{\odot}$ are sparse, and require speeds of $\sim 1000 \mathrm{~km} / \mathrm{s}$. Only systems with masses as low as $M \approx 10^{6-7} \mathrm{M}_{\odot}$ (lowest shaded curve) are sufficiently abundant to widely disperse metals with outflow speeds of a few $\times 10 \mathrm{~km} / \mathrm{s}$. However, such low-mass systems have velocity dispersions below $\sigma=10 \mathrm{~km} / \mathrm{s}$, and are unlikely to have formed metals in the presence of the post-reionization UV background (the horizontal solid curve shows the halo mass corresponding to $\sigma=10 \mathrm{~km} / \mathrm{s}$ ). The natural conclusion is that the metals had to be produced and dispersed by low-mass systems, $M \approx 10^{6} \mathrm{M}_{\odot}$, prior to reionization at $z \gtrsim 6.3$.

\subsection{The Growth of Supermassive Black Holes}

A third line of evidence for high-redshift activity comes from the sheer size of supermassive black holes (BHs) required to power the recently discovered 
bright quasars near $z \approx 6$. Assuming that these quasars are shining at their Eddington limit, and are not beamed or lensed], their BH masses are inferred to be $M_{\mathrm{bh}} \sim 4 \times 10^{9} \mathrm{M}_{\odot}$. The Eddington-limited growth of these supermassive BHs by gas accretion onto stellar-mass seed holes, with a radiative efficiency of $\epsilon \equiv L / \dot{m} c^{2} \approx 10 \%$, requires $\sim 20$ e-foldings on a timescale of $t_{\mathrm{E}} \sim 4 \times 10^{7}(\epsilon / 0.1)$ years. While the age of the universe leaves just enough time $\left(\lesssim 10^{9}\right.$ years $)$ to accomplish this growth by redshift $z=6$, it does mean that accretion has to start early, and the seeds for the accretion have to be present at ultra-high redshifts: $z \gtrsim 15(20)$ for an initial seed mass of $100(10) \mathrm{M}_{\odot}$. Furthermore, the radiative efficiency cannot be much higher than $\epsilon \approx 10 \%[13,14$. Since an individual quasar $\mathrm{BH}$ could have accreted exceptionally fast (exceeding the Eddington limit), it will be important to apply this argument to a larger sample of high-redshift quasars. Nevertheless, we note that a comparison of the light output of quasars at the peak of their activity $(z \sim 2.5)$ and the total masses of their remnant BHs at $z=0$ shows that during the growth of most of the $\mathrm{BH}$ mass the radiative efficiency cannot be much smaller than 10\%, and hence any 'super-Eddington' phase must be typically restricted to building only a small fraction of the final $\mathrm{BH}$ mass.

\section{The Assembly of the First Galaxies}

While the formation of non-linear dark matter halos can be followed from firstprinciples, the formation of stars or BHs in these halos is much more difficult to model ab-initio. Nevertheless, we may identify three important mass-scales, which collapse at successively smaller redshifts,

1. Gas can only condense in dark halos above the cosmological Jeans mass, $M_{\mathrm{J}} \approx 10^{4} \mathrm{M}_{\odot}[(1+\mathrm{z}) / 11]^{3 / 2}$, so that the gravity of dark matter can overwhelm gas pressure in the IGM.

2. Gas that condensed into Jeans-unstable halos can cool and contract further in halos with masses above $M_{\mathrm{H} 2} \gtrsim 10^{5} \mathrm{M}_{\odot}[(1+\mathrm{z}) / 11]^{-3 / 2}$ (virial temperatures of $T_{\mathrm{vir}} \gtrsim 10^{2} \mathrm{~K}$ ), provided there is a sufficient abundance of $\mathrm{H}_{2}$ molecules (at a level of at least $\left.n_{\mathrm{H} 2} / n_{\mathrm{H}} \sim 10^{-3}\right)$.

3. In halos with masses above $M_{\mathrm{H}} \gtrsim 10^{8} \mathrm{M}_{\odot}[(1+\mathrm{z}) / 11]^{-3 / 2}$ (virial temperatures of $T_{\text {vir }} \gtrsim 10^{4} \mathrm{~K}$ ), gas can cool and contract via excitation of atomic Ly $\alpha$, even in the absence of any $\mathrm{H}_{2}$.

The first stars or BHs likely formed in gas that cooled and condensed via excitations of roto-vibrational levels of $\mathrm{H}_{2}$ molecules in dark matter condensations with $M \sim 10^{5} \mathrm{M}_{\odot}$ at redshift $z \sim 25$ (corresponding to $\sim 3 \sigma$ peaks in the primordial density field) [15]16]. Unless the first rare objects were significant sources of X-ray photons with energies $E \gtrsim 1 \mathrm{keV}$, their soft UV radiation, permeating the distant universe at photon energies $E<13.6 \mathrm{eV}$, caused a negative feedback,

\footnotetext{
${ }^{2}$ Strong lensing or beaming would contradict the large proximity effect around these quasars [12].
} 


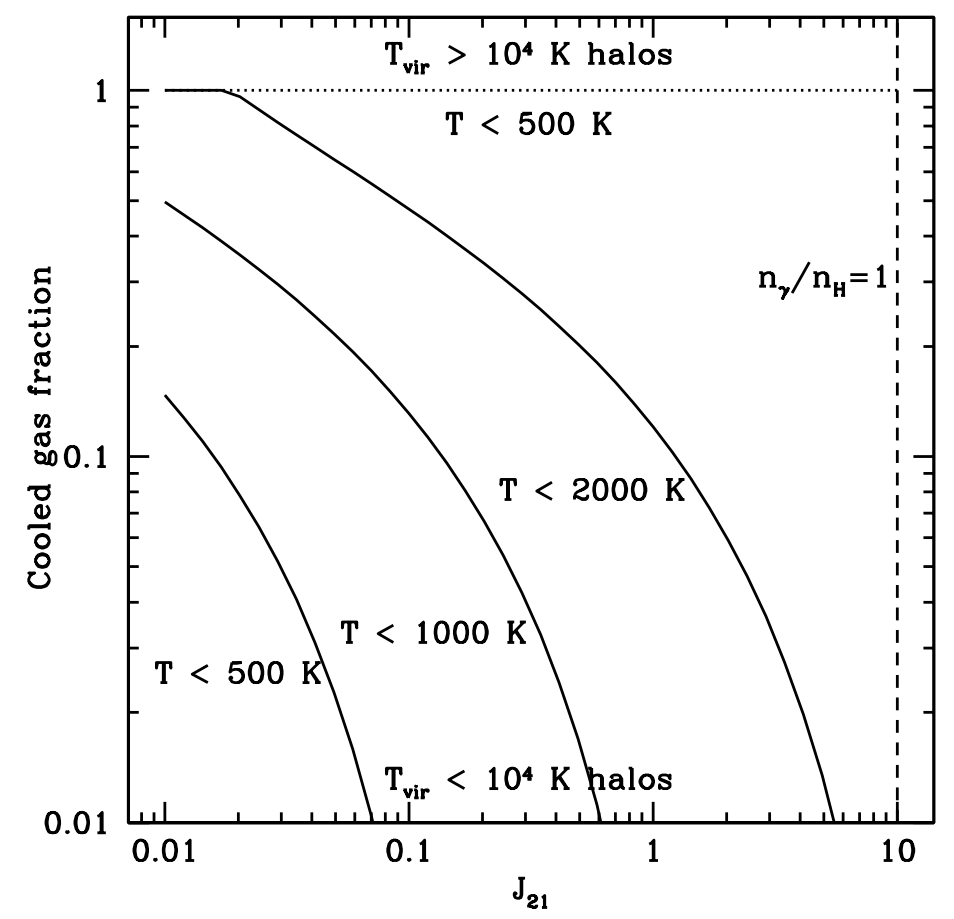

Fig. 3. The fraction of gas in a halo that can cool below a given temperature $T$, as a function of the external UV radiation field $J_{21}$, for halos at $\mathrm{z}=15$. In halos with $T_{\text {vir }}<10^{4} \mathrm{~K}$ (lower three solid curves), only a small fraction of the gas is at sufficiently high density to be unaffected by external radiation fields and cool. By contrast, in halos with $T_{\text {vir }}>10^{4} \mathrm{~K}$, where atomic cooling operates, the gas contracts to high densities, and virtually all of the disk gas can form $\mathrm{H}_{2}$ and cool to low temperatures (dotted horizontal line). The UV intensity corresponding to one ionizing photon per baryon in the universe is marked by the dashed vertical line on the right. Adopted from [19].

strongly suppressing $\mathrm{H}_{2}$ cooling and star-formation in clumps that collapsed subsequently [17, 18. Efficient and widespread star (and/or BH) formation, capable of reionizing the universe, had to then await the collapse of halos with $T_{\text {vir }}>10^{4} \mathrm{~K}$, or $M_{\text {halo }}>10^{8}[(1+z) / 11]^{-3 / 2} \mathrm{M}_{\odot}$.

The evolution of halos with $T_{\text {vir }}>10^{4} \mathrm{~K}$ differs from their less massive counterparts [19]. Efficient atomic line radiation allows rapid cooling to $\sim 8000 \mathrm{~K}$; subsequently the gas can contract to high densities nearly isothermally at this temperature. In the absence of $\mathrm{H}_{2}$ molecules, the gas would likely settle into a locally stable disk and only disks with unusually low spin would be unstable. However, the initial atomic line cooling leaves a large, out-of-equilibrium residual free electron fraction. This allows the molecular fraction to build up to a universal value of $x_{\mathrm{H}_{2}} \approx 10^{-3}$, almost independently of initial density and tem- 
perature (this is a non-equilibrium freeze-out value that can be understood in terms of timescale arguments [19]).

Unlike in less massive halos, $\mathrm{H}_{2}$ formation and cooling is much less susceptible to feedback from external UV fields. This is because the high densities $n$ that can be reached via atomic cooling. The $\mathrm{H}_{2}$ abundance that can build up in the presence of a UV radiation field $J_{21}$, and hence the temperature to which the gas will cool, is controlled by the ratio $J_{21} / n$. For example, in order for a parcel of gas to cool down to a temperature of $500 \mathrm{~K}$, this ratio has to be less than $\sim 10^{-3}$ (where $J_{21}$ has units of $10^{-21} \mathrm{erg} \mathrm{s}^{-1} \mathrm{~cm}^{-2} \mathrm{~Hz}^{-1} \mathrm{sr}^{-1}$, and $n$ has units of $\mathrm{cm}^{-3}$ ). In Figure 3, we show, as a function of the external UV radiation field $J_{21}$, the mass fraction of gas which is able to cool to a temperature $T$ in $T_{\text {vir }}<10^{4} \mathrm{~K}$ halos (the gas is assumed to be in hydrostatic equilibrium within an NFW halo [20]), and $T_{\text {vir }}>10^{4} \mathrm{~K}$ halos (the gas is assumed to have cooled via atomic Ly $\alpha$ to a $10^{4} \mathrm{~K}$, rotationally supported disk 21]).

The figure reveals that flux levels well below that required to fully reionize the universe strongly suppresses the cold gas fraction in $T_{\text {vir }}<10^{4} \mathrm{~K}$ halos. By comparison, the UV flux has nearly negligible impact on $\mathrm{H}_{2}$ formation and cooling in $T_{\text {vir }}>10^{4} \mathrm{~K}$ halos, where all of the gas is able to cool to $T=500 \mathrm{~K}$. Indeed, under realistic assumptions, the newly formed molecules in the dense disk can cool the gas to $\sim 100 \mathrm{~K}$, and allow the gas to fragment on scales of a few $\times 100 \mathrm{M}_{\odot}$. Various feedback effects, such as $\mathrm{H}_{2}$ photodissociation from internal UV fields, and radiation pressure due to Ly $\alpha$ photon trapping, are then likely to regulate the eventual efficiency of star formation in these systems.

\section{Future Observational Signatures}

Although the first generation of galaxies are distant, and intrinsically faint objects, they should be within reach of NGST. If $\sim 10 \%$ of the gas turns into stars in a $10^{8} \mathrm{M}_{\odot}$ halo, it should be detectable in the $1-5 \mu \mathrm{m}$ band with $N G S T$ out to redshifts beyond $z=10$ in a $10^{4}$ second integration. $\mathrm{A} \sim 10^{5} \mathrm{M}_{\odot}$ black hole, forming out of $\sim 1 \%$ of the gas in such a halo, and shining at its Eddington limit, would be detectable to a similar redshift. Simple "semi-analytical" models 22,23. predict that NGST will either detect a significant number of ultra-high redshift sources, or else it will severely constraint any early model of early structure formation. Similarly optimistic conclusions can be drawn about detecting recombinant line emission from high-redshift sources: star formation rates as small as $\sim 1 \mathrm{M}_{\odot} / \mathrm{yr}$, or BHs as small as $\sim 10^{5} \mathrm{M}_{\odot}$ translate into detectable $\mathrm{H} \alpha$ and He line fluxes beyond $z=10$, as long as the escape fraction of $\mathrm{H}-$ and $\mathrm{He}-$ ionizing radiation from these sources is low 24. Finally, as the baryons cool and contract inside high-redshift halos with virial temperatures $T \gtrsim 10^{4} \mathrm{~K}$, they likely channel a significant fraction of their gravitational binding energy into the Ly $\alpha$ line. At the limiting line flux $\approx 10^{-19} \mathrm{erg} \mathrm{s}^{-1} \mathrm{~cm}^{-2} \mathrm{asec}^{-2}$ of the NGST, several sufficiently massive halos, with velocity dispersions $\sigma \gtrsim 120 \mathrm{~km} \mathrm{~s}^{-1}$, would be visible per $4^{\prime} \times 4^{\prime}$ field. The halos would have characteristic angular sizes of $\sim 10^{\prime \prime}$, and could be detectable in a broad-band survey out to $z \approx 6-8$ 
(but not beyond the reionization redshift, where Ly $\alpha$ photons are be resonantly scattered). Their detection would provide a novel and direct probe of galaxies caught in the process of their formation 25:26- possibly before the first stars or quasars even lit up.

I thank the organizers of this workshop for their kind invitation, and Peng Oh for many recent fruitful discussions, and for permission to draw on our joint work. I acknowledge support from NASA through a Hubble Fellowship.

\section{References}

1. W.H. Press, P.L. Schechter: ApJ, 181, 425 (1974)

2. R.K. Sheth, H.J. Mo, G. Tormen: MNRAS, 323, 1 (2001)

3. A. Jenkins, et al.: MNRAS, 321, 372 (2001)

4. R.H. Becker et al.: AJ, in press, preprint astro-ph/0108097 (2001)

5. P. Madau: Physica Scripta, volume T, 85, 156 (2000)

6. Z. Haiman, T. Abel, P. Madau: ApJ, 551, 599 (2001)

7. P.R. Shapiro, A.C. Raga, G. Mellema: in Molecular Hydrogen in the Early Universe, Memorie Della Societa Astronomica Italiana, Vol. 69, ed. E. Corbelli, D. Galli, and F. Palla (Florence: Soc. Ast. Italiana), p. 463

8. R. Barkana, A. Loeb: ApJ, 523, 54 (1999)

9. S.L. Ellison, A. Songaila, J. Schaye, M. Pettini: AJ, 120, 1167 (2000)

10. A. Aguirre, L. Hernquist, J. Schaye, D.H. Weinberg, N. Katz, J. Gardner: ApJ, 560, 599 (2001)

11. P. Madau, A. Ferrara, M.J. Rees: ApJ, 555, 92 (2001)

12. Z. Haiman, R. Cen: in preparation, to be submitted to ApJ

13. Z. Haiman, A. Loeb: ApJ, 552, 459 (2001)

14. R. Barkana, Z. Haiman, J.P. Ostriker: ApJ, 558, 482 (2001)

15. T. Abel, G.L. Bryan, M.L. Norman: ApJ, 540, 39 (2000)

16. V. Bromm, P. Coppi, R.B. Larson: ApJ, 527, 5 (1999)

17. Z. Haiman, T. Abel, M.J. Rees: ApJ, 534, 11 (2000)

18. M.E. Machacek, G.L. Bryan, T. Abel: ApJ, 548, 509 (2001)

19. S.P. Oh, Z. Haiman: ApJ, submitted, preprint astro-ph/0108071 (2001)

20. J.F. Navarro, C.S. Frenk, S.D.M. White: ApJ, 490, 493 (1997)

21. H.J. Mo, S. Mao, S.D.M. White: MNRAS, 295, 319 (1998)

22. Z. Haiman, A. Loeb: ApJ, 503, 505 (1998)

23. Z. Haiman, D.N. Spergel, E.L. Turner: ApJ, submitted, preprint astro-ph/0110226 (2001)

24. S. P. Oh, Z. Haiman, M. Rees: ApJ, 553, 73 (2001)

25. Z. Haiman, M. Spaans, E. Quataert: ApJ, 537, L9 (2000)

26. Z. Haiman, M.J. Rees: ApJ, 556, 87 (2001) 